\title{
Les langues d'enseignement entre politiques officielles et stratégies des acteurs
}

Introduction

\section{Abdeljalil Akkari et Daniel Coste}

\section{(Q) OpenEdition}

\section{Journals}

Édition électronique

URL : https://journals.openedition.org/ries/4487

DOI : $10.4000 /$ ries. 4487

ISSN : 2261-4265

\section{Éditeur}

France Education international

Édition imprimée

Date de publication : 1 décembre 2015

Pagination : 41-52

ISSN : 1254-4590

Référence électronique

Abdeljalil Akkari et Daniel Coste, « Les langues d'enseignement entre politiques officielles et stratégies des acteurs », Revue internationale d'éducation de Sèvres [En ligne], 70 | décembre 2015, mis en ligne le 18 janvier 2015, consulté le 01 juillet 2021. URL : http://journals.openedition.org/ries/4487 ; DOI https://doi.org/10.4000/ries.4487 


\title{
Les langues d'enseignement, un enjeu politique
}

\author{
Les langues \\ d'enseignement entre \\ politiques officielles et \\ stratégies des acteurs
}

\section{Introduction}

\author{
Abdeljalil Akkari \\ Université de Genève \\ Daniel Coste \\ École normale supérieure de Lyon
}

Le choix de la langue ou des langues utilisées pour enseigner a toujours été, dans les différents systèmes éducatifs, un enjeu éminemment politique. Longtemps abordée sous le seul angle des politiques étatiques nationales, cette question subit aujourd'hui l'influence de facteurs et acteurs multiples et plus ou moins présents suivant les contextes : perspectives d'employabilité et de circulation professionnelle, obligation de résultats et de reddition des comptes, privatisation et diversification de l'offre de formation, jeu des stratégies familiales, internationalisation des politiques éducatives, mais aussi, selon les cas, réaffirmations monolingues centralisatrices ou reconnaissance de langues minorisées, minoritaires ou régionales dans la scolarisation. Question plus que jamais politique donc, mais les décisions sont le fait d'acteurs divers, tant privés que publics, et peuvent différer selon les filières et niveaux du cursus, de l'école enfantine à l'enseignement supérieur. Les contributions rassemblées dans ce dossier 70 de la Revue internationale d'éducation de Sèvres rappellent aussi l'incidence durable de facteurs historiques et géopolitiques et les tensions internes qui peuvent en résulter.

Dans un contexte donné, si le choix répond toujours à des considérations de nature politique, il s'effectue notamment par l'intermédiaire de l'arsenal législatif dont disposent l'État et les pouvoirs publics, arsenal qui s'étend de la Constitution d'un pays, aux lois, aux règlements et aux directives particulières. Dans les régimes pluralistes, le parlement est une arène particulièrement importante pour une plus grande ouverture ou une fermeture envers les langues. Ensuite, une fois que le politique a fixé le cadre et l'orientation, à chaque niveau du système 
éducatif, des ministères de l'éducation jusqu'aux classes dans les établissements scolaires, les politiques éducatives quant à la langue d'instruction sont interprétées, négociées et reconstruites dans un processus complexe d'opérationnalisation et d'application. En dépit de cette complexité, la plupart des recherches ciblant les politiques éducatives des langues demeurent axées sur le niveau national, n'analysant le plus souvent que les documents officiels. Les articles de ce numéro montrent qu'une attention particulière doit être accordée aux résistances locales et à l'analyse de la manière dont les acteurs, en particulier les élites politiques, les enseignants et les parents, traduisent ou/et contournent les orientations officielles. Autrement dit, l'analyse des politiques éducatives au niveau national doit être complétée par l'exploration des relectures et révisions locales.

Le statut de la langue d'instruction dans les systèmes éducatifs a longtemps été reconnu non seulement comme un indicateur très important des relations de pouvoir dans les sociétés, mais aussi comme un instrument crucial pour la continuité et/ou le changement à l'école (Bray \& Koo, 2004).

\section{Affirmation IDENTITAIRE ET « POIDS » DES LANGUES}

Nombre d'États-nations se sont construits ou reconstruits en donnant à une ou plusieurs langues nationales une place symbolique et centrale dans leur affirmation identitaire et unitaire. À l'épreuve de la globalisation, des migrations, des dynamiques régionales, ces langues nationales peuvent se voir disputer, ne serait-ce qu'à la marge et par certains segments de leur population, leur rôle dans la scolarisation. Les politiques qui visent le renforcement de l'identité nationale par la réduction réglementée de la diversité linguistique s'avèrent ainsi de plus en plus irréalistes. Comme le souligne Lippi-Green (1997), vouloir que tous les individus parlent la même langue est aussi chimérique qu'exiger qu'ils aient tous la même taille.

Au demeurant, dans bien des pays, les politiques de décentralisation et de privatisation donnent une marge de manœuvre plus grande aux acteurs locaux de l'éducation pour infléchir la politique traditionnelle associant identité nationale et langue d'instruction unique ou officielle. Dans de nombreux États fédéraux, la gouvernance décentralisée de l'éducation permet aux pouvoirs locaux d'utiliser la valorisation des langues locales comme une ressource pour accroître leur légitimité. Évidemment, les tensions peuvent être très différentes selon la nature et l'ancienneté de l'organisation de l'État fédéral. Ainsi, la politique linguistique de la Suisse semble apaisée en matière de choix de la langue d'instruction, en raison du principe de territorialité. Autrement dit, le choix de la langue d'instruction est lié à la langue reconnue comme dominante dans un territoire ou un canton du pays. En Espagne en revanche, le choix de la langue d'instruction est plus disputé, en raison de l'enchevêtrement des territoires linguistiques et des projets d'indépendance de certaines régions du pays. 
En ce qui concerne les parents, ce sont la valeur de la langue dans la lutte pour les places de travail et la mobilité sociale qui vont le plus souvent déterminer leurs stratégies. L'anglais ou d'autres langues prestigieuses surgissent alors comme langue dominante d'instruction dans le réseau privé ou voient leur place renforcée dans le réseau public, à la demande des familles. Paradoxalement, la connotation coloniale rattachée à l'anglais et à d'autres langues européennes semble s'estomper dans les pays du Sud, à la faveur d'une mondialisation économique et culturelle dans laquelle l'anglais ou d'autres langues peuvent jouer un rôle majeur. Ainsi, le chinois gagne de l'espace en Asie du Sud-Est, en raison de l'expansion économique de la Chine, comme le montre par exemple, dans cette livraison, l'article d'Aishah Binte Mohamad Kassim consacré à Singapour. Dans un autre registre, des écoles franco-arabes prospèrent un peu partout en Afrique de l'Ouest, avec la modernité rattachée au français et l'identité religieuse accrue permise par l'arabe.

À considérer les pays représentés dans le présent recueil et s'agissant d'affirmation identitaire, il est intéressant d'observer des contextes où s'exerce une volonté politique forte de restauration nationale (comme dans le cas de l'Estonie, que présente Martin Ehala) ou d'autonomie régionale (comme pour le Pays basque espagnol, caractérisé par Beñat Muguruza), voire de tradition d'intégration de populations allophones ou de défense de positions internationales, s'agissant de la France. C'est là, en particulier, qu'intervient le "poids » relatif des langues (Calvet et Calvet, 2013). Dans la situation des pays baltes, on a affaire à des langues (lituanien, letton, estonien) de faible diffusion internationale et longtemps minorées, voire réprimées par des occupations étrangères anciennes et plus récentes. Pour le Pays basque, la langue est typologiquement isolée et la population bascophone est aussi hispanophone ; le volontarisme politique local et une offre éducative proposant aux familles plusieurs formules curriculaires a permis le développement de la scolarisation en basque, avec l'appui d'une majorité des familles. La position du français en France (analysée ici par Gérard Vigner) est évidemment de tout autre nature, mais une longue tradition centralisatrice et normative en fait aussi une langue à forte charge identitaire et à dimension internationale affirmée, enjeu de débats sociaux et médiatiques, marquée par une relation ambivalente avec les langues régionales et avec la montée de l'anglais, aussi bien qu'avec la persistance, voire le développement, des langues de la migration.

À bien des égards, mais de façon peut-être paradoxale, l'ambivalence marque aussi la politique linguistique algérienne dans sa continuité et ses fluctuations depuis l'indépendance du pays. Langue majeure et de " poids » certain au niveau international, l'arabe est seule langue nationale officielle, constamment réaffirmée comme telle, porteuse de l'identité algérienne, mais, à des titres très divers, la place du français, première langue étrangère, celle du tamazight, celle de la darija (parlers dialectaux) viennent, ainsi que le marque la contribution de Khaoula Taleb Ibrahimi, singulièrement complexifier le paysage linguistique, alimenter le débat public et diversifier les stratégies des acteurs sociaux. 
Un indicateur notable des rapports entre langues dans des pays ou régions marqués par l'affirmation centrale forte d'une langue à valeur identitaire posée comme commune se situe au niveau de l'enseignement supérieur. Dans les pays du Maghreb, même s'il recule de façon notable devant l'anglais au niveau de la recherche, le français reste langue d'enseignement dans plusieurs secteurs du système universitaire, notamment dans les filières comme les sciences exactes ou la médecine. Les formations universitaires de la communauté autonome du Pays basque assurent des enseignements en basque (histoire, géographie, philosophie, éducation) mais fonctionnent pour l'essentiel en espagnol, à des fins aussi d'ouverture internationale. En Estonie, la plus grande université estonienne, l'Université de Tartu, comptait en 2012 cinquante-cinq programmes de doctorat en langue anglaise contre seulement quatre en langue estonienne.

\section{MINORITÉS INTERNES ET FORMES D'ÉDUCATION BILINGUE OU PLURILINGUE}

Dans certains contextes multilingues, et notamment dans des pays d'Afrique sub-saharienne ou d'Asie où ont prévalu les langues des anciens colonisateurs, les politiques officielles peuvent stipuler pour l'instruction scolaire l'usage des langues maternelles ou premières ayant aujourd'hui statut de langues officielles et/ou nationales. Cette volonté se heurte souvent à des difficultés d'ordres divers (financiers, humains, symboliques ou pratiques...) et peut rencontrer la résistance de certains parents, à même de se tourner vers des dispositifs autres - et notamment privés - où des langues perçues comme à plus grande valeur ajoutée sont langues de scolarisation. Ces situations, et les expériences auxquelles elles donnent lieu, ont fait l'objet d'études diverses. On trouvera dans le présent recueil, avec l'article d'Alkassoum Maiga, Abou Napon et Zakaria Sore, un écho particulier d'enquêtes menées au Burkina Faso et faisant apparaître le clivage entre secteur formel et secteur informel.

Plus généralement et dans d'autres contextes, le choix de la ou des langue(s) d'instruction peut résulter d'un processus, parfois conflictuel, de décentralisation ou d'autonomie de minorités nationales ou régionales présentant des spécificités historiques, ethniques ou politiques. Des secteurs du système éducatif opèrent (en tout ou en partie) dans une langue autre que la ou les langue(s) nationale(s) et/ou officielle(s) du pays, cela avec des formes et degrés variables d'enseignement bilingue ou plurilingue. On songera ici, par exemple, aux situations du Pays basque espagnol, déjà mentionné, de la Catalogne, du Val d'Aoste, mais aussi à celles des minorités polonophones ou russophones de Lituanie, russophones de Lettonie et d'Estonie ou encore, à une tout autre échelle, à celle, qui fait l'objet ici d'une contribution de Padma Sarangapani, des États et Territoires de l'Inde. On peut aussi citer les expériences d'instruction en langues maternelles pour les populations indigènes mises en œuvre et parfois 
développées à l'échelle régionale un peu partout en Amérique latine, du Mexique au Chili. Le cas du Paraguay est à ce propos très instructif, comme le montre la contribution de Dominique Demelenne. Dans ce pays, malgré la bienveillance politique initiée par la progressive démocratisation du début des années 1990, la prise en compte du guarani comme langue d'instruction dans les expériences d'éducation bilingue est ardue. Elle rencontre une forte réticence chez les cadres du ministère de l'éducation et le scepticisme des parents.

La possibilité de la promotion d'un véritable plurilinguisme scolaire se heurte souvent à divers facteurs mis en évidence par la recherche récente.

En premier lieu, il existe une résistance dans les institutions publiques, qui ont fonctionné pendant longtemps dans la langue des classes dominantes, notamment dans les contextes postcoloniaux. Comme le souligne Desai (2013) à propos de l'Afrique, les langues locales sont bonnes pour le marché mais pas pour l'école. L'exemple de l'Afrique du Sud postapartheid est révélateur. Malgré la réforme de 1997, les dispositifs actuels continuent de privilégier les élèves parlant l'anglais et l'afrikaner. Les pratiques linguistiques locales ne peuvent pas changer la dynamique du pouvoir dans des sociétés qui restent inégalitaires. C'est seulement quand les langues sont effectivement utilisées dans des institutions valorisées, telles que l'école mais aussi d'autres, qu'elles se développent pleinement. Cette même ambiguïté des politiques publiques a été mise en évidence au Sénégal. Selon Cissé (2005), le Sénégal est un pays multilingue et multiculturel que la politique linguistique a réduit à un monolinguisme ou, au mieux, à un bilinguisme français/langues nationales. L'introduction des langues nationales dans l'enseignement formel n'aura de sens que lorsqu'elle sera suivie d'une volonté d'assigner à chacune d'elles le rôle social, économique et politique qui lui revient. La politique de réglementation de l'aménagement linguistique par décrets se heurte toujours, sur le terrain, à divers obstacles, parce qu'elle ne contribue qu'à élargir le fossé entre des discours de principe et des pratiques contradictoires. L'État invoque souvent le manque de moyens matériels et humains. Mais c'est bien le manque de volonté politique de la part des autorités qui reste le facteur le plus décisif dans le maintien de la prédominance de la langue de l'ancien colonisateur comme langue d'instruction.

Dans le secteur de l'éducation non formelle et de l'alphabétisation, le pluralisme linguistique semble plus présent, comme le montre, dans ce dossier, le cas déjà mentionné du Burkina Faso. Néanmoins, toutes les langues n’ont pas la même présence dans les cours d'alphabétisation.

En second lieu, il est important de réfléchir aux problèmes d'application des réformes visant à promouvoir l'usage des langues maternelles dans l'enseignement. Le cas du Népal est à cet égard édifiant. Une politique linguistique bien intentionnée visant à promouvoir la diversité linguistique et à répondre aux difficultés d'apprentissage que rencontrent les enfants des minorités linguistiques peut faire face à une résistance idéologique et de mise en œuvre sur le terrain. Les communautés autochtones et les parents sont souvent ambivalents 
ou même réticents quant à la pertinence de l'usage de leurs propres langues à l'école (Skattum, 2010 ; Phyak, 2013). Par conséquent, il est indispensable d'intégrer la langue facilitant la mobilité sociale dans toute politique des langues à l'école (Grandguillaume, 2009). Le cas de l'enseignement bilingue au Paraguay, évoqué plus haut, demande réflexion dans cette perspective ; tout comme, à d'autres égards, celui de l'éducation de base aux Comores, qu'examine Aurélie Chauvet.

\section{Mesure, Culte de l'excellence ET ÉDUCATION DU CITOYEN DANS UNE SOCIÉTÉ HÉTÉROGÈNE}

Les évaluations internationales, les besoins de l'économie peuvent conduire à une segmentation de réseaux éducatifs parallèles et inégalitaires, notamment quant au rapport à l'emploi. Le choix de la ou des langue(s) d'enseignement relève ainsi du défi d'adapter les systèmes éducatifs aux visées hétérogènes des parents et aux pressions liées à la globalisation, tout en contribuant à la formation de citoyens d'une société plurielle.

Les pays de l'Asie représentent à cet égard un cas intéressant, dans la mesure où, de plus en plus, leurs systèmes éducatifs sont présentés comme exemplaires à la fois pour les pays du Sud mais aussi, plus récemment, pour les pays de l'OCDE. Un impact majeur de la mondialisation sur l'éducation en Asie est la tendance croissante à utiliser l'anglais comme langue d'instruction. Le cas de Singapour, auquel l'article d'Aishah Binte Mohamad Kassim est ici consacré, semble particulièrement révélateur du rapport entre langues communautaires et langue d'une certaine mondialisation. Bien que la région de l'Asie, dans son ensemble, reflète la mondialisation de l'anglais comme moyen d'instruction, les politiques éducatives des pays de la région peuvent avoir des motivations, des objectifs et des stratégies spécifiques pour protéger leurs propres intérêts et identités nationales. Plus important encore, au niveau macro-politique, il semble que se développe une compréhension simpliste de l'anglais, langue d'instruction, comme solution peu coûteuse à des problèmes linguistiques complexes, s'agissant d'atteindre des objectifs politico-économiques très ambitieux (Hamid, Nguyen \& Baldauf Jr, 2013).

L'article consacré à l'Inde dans ce dossier met en évidence la complexité du statut de l'anglais dans le système éducatif de ce pays. Si la Constitution affirme le droit de l'enfant à être instruit dans sa langue maternelle, les pratiques et les choix des langues d'instruction placent l'anglais au centre de l'échiquier politique. De fait, le marché scolaire et certains pouvoirs régionaux remettent en question les principes inscrits dans la Constitution.

L'utilisation de l'anglais ou d'autres langues étrangères comme langue d'instruction aboutit à une structure du système scolaire en réseaux à plusieurs vitesses et de qualité hétérogène. L'écart entre les élèves selon leur appartenance 
sociale se creuse, dans les épreuves basées sur des tests standardisés. Grâce aux ressources supplémentaires et aux cours particuliers, appelés par Bray (2009) "l'éducation de l'ombre » ou shadow education, un groupe plus ou moins grand d'élèves issus de familles favorisées excellera dans le système, tandis que la plupart des autres jeunes restera à la marge. Cette situation contribue fortement à l'aggravation de la pauvreté pour les classes défavorisées. Une éducation de qualité pour tous ne peut se construire sans que soit pris en compte sérieusement le problème de la langue d'instruction (Brock-Utne, 2007).

Il est par conséquent important de mettre en évidence les rapports entre langue, pouvoir et inégalité. Par ailleurs, il est pertinent d'avoir une lecture critique des politiques linguistiques, c'est-à-dire de comprendre les implications politiques et sociales de politiques particulières adoptées dans des contextes historiques spécifiques. Les politiques linguistiques affectent la vie de toute la société, et tout particulièrement celle d'individus et de groupes qui ont souvent peu d'influence sur le processus de construction des politiques publiques (Tollefson, 2002 ; Menken \& García, 2010).

Abordée dans ce volume par la contribution de Jean-Claude Beacco, l'action des organisations internationales telles que le Conseil de l'Europe peut s'avérer utile dans la mesure où, par le biais notamment de recommandations et de documents de référence, elle interroge les traditions conservatrices en matière d'ouverture linguistique. Par l'attention portée aux langues minoritaires ou régionales, le Conseil de l'Europe a contribué à la prise en compte de la diversité linguistique et culturelle ; et, complémentairement, son Unité des politiques linguistiques a produit des guides et manuels centrés sur les langues de scolarisation dans leur rapport à la qualité et à l'équité de l'éducation.

\section{UNE DIVERSITÉ DE SITUATIONS}

La situation de l'Algérie, telle que décrite par Khaoula Taleb Ibrahimi est marquée par le passé de la colonisation et par les différentes phases de la politique d'arabisation de l'école fondamentale depuis l'indépendance du pays. La scolarisation peine encore à garantir aux élèves, en fin d'études secondaires, un niveau satisfaisant d'expression écrite en langue nationale. La maîtrise du français, première langue étrangère, reste généralement insuffisante pour un bon accès à celles des filières universitaires où le français est langue de travail. La distance entre l'arabe comme langue majeure de scolarisation et le répertoire des élèves pratiquant l'arabe dialectal et/ou le tamazight continue à faire difficulté et le système public est contourné par nombre de familles des milieux dirigeants ou des élites locales. D’où le recours à des établissements privés (et payants), où le français tient une place plus importante et qui, après être restés un temps en marge clandestine, bénéficient aujourd'hui d'une forme conventionnelle de reconnaissance officielle. 
L'article d'Alkassoum Maiga, Abou Napon et Zakaria Soré porte sur le contexte fondamentalement multilingue du Burkina Faso. Ce texte met en évidence les différences importantes dans l'utilisation des langues entre les secteurs de l'éducation formelle et de l'éducation non formelle. Dans le premier secteur, malgré la diversité linguistique du pays, l'éducation bilingue reste à un stade expérimental et le français demeure la principale langue d'instruction. L'éducation bilingue a été, durant les dernières décennies, dépendante des changements en matière de politiques linguistiques opérés par le gouvernement mais aussi de l'appui récent de la coopération internationale. Dans le non formel, les enseignements sont essentiellement dispensés en langues nationales. En dépit de leur marginalisation dans le système formel d'éducation, la soixantaine de langues nationales, en particulier les trois principales (mooré, le fulfudé (ou peul) et le dioula), sont bien présentes dans la vie quotidienne des burkinabais ainsi que le mettent en évidence les enquêtes menées sur les différents terrains.

La contribution d'Aurelie Chauvet est consacrée aux statuts des langues dans l'éducation de base aux Comores. L'auteur montre la cohabitation nécessaire de trois langues aux statuts et fonctions différents tout au long de l'éducation de base. Le shikomori comme langue première représente une langue essentielle dans le préscolaire. Il doit être maintenu tout le long de la scolarité primaire, à égalité avec le français, qui est introduit progressivement dans ce cycle. Se pose ici la problématique de la valeur accordée par l'État et les parents à ces deux langues. Autrement dit, comment ne pas cantonner le shikomori à un rôle de transition vers le français et éviter que ce dernier ne monopolise les possibilités de mobilité sociale. L'arabe, langue de la socialisation religieuse, peut, pour sa part, faciliter l'entrée de l'enfant comorien dans la culture de l'écrit et lui donner accès à une langue internationale ayant eu une présence historique importante sur toute la côte orientale de l'Afrique.

Martin Ehala analyse dans son article le façonnage de l'écologie des langues en Estonie. Ce pays a connu des changements continus en matière de politique linguistique durant la période postsoviétique. Sur le plan politique, les initiatives diverses ont visé à " garantir la préservation de la nation, de la langue et de la culture de l'Estonie à travers les âges ", pour reprendre le préambule de la Constitution. Les stratégies portent notamment sur la défense et illustration de la langue estonienne, longtemps minorée, et dont la préservation et l'usage généralisé passent d'abord par la scolarisation. Les initiatives des autorités centrales sont entravées à la fois par la présence de locuteurs russophones, minoritaires à l'échelle nationale mais majoritaires dans certaines régions, mais aussi par l'émergence d'une forte demande sociale vers l'anglais, dans une Estonie qui cherche sa place dans l'Union européenne et la mondialisation.

Le texte de Gérard Vigner montre que le français, si fermement installé en France en tant que langue d'instruction, objet de discours et de débats dans la société, fortement normé dans ses usages scolaires, ne peut qu'être affecté par 
les évolutions du monde. La construction de l'Union européenne et le développement de nombreux courants migratoires tendant à déstabiliser l'équilibre général entre les langues, installé de longue date dans le pays. La question qui est posée au français n'est plus celle de l'articulation du local et du national, comme au temps de la Troisième République, mais d'un national avec un local revisité (les autres langues de France, y compris les langues de la migration et celles de l'outre-mer), d'un national aux prises avec des courants transnationaux de plus en plus présents sur le territoire.

L'article de Padma Sarangapani aborde le débat actuel sur la langue d'instruction en Inde. Il met en évidence les contradictions et les hésitations législatives dans ce domaine. Le débat en Inde se cristallise autour d'une tension permanente entre "annonces des principes " par le législateur du droit à l'éducation en langue maternelle et "stratégies de contournement » de la législation par différents acteurs. La récente Loi sur le droit à l'éducation (2009) fait écho à la Constitution en affirmant que « la langue d'enseignement doit, dans la mesure du possible, être la langue maternelle de l'enfant " (Gouvernement de l'Inde, 2009). Mais, en même temps, en 2013, la Cour suprême de l'Inde a déclaré que le choix de la langue d'enseignement devait être laissé aux parents et que l'État ne pouvait pas contraindre les élèves à étudier seulement dans leur langue maternelle. Les parents jouent un rôle important dans les initiatives visant à proposer l'anglais comme langue d'instruction pour différents groupes sociaux. De surcroît, des considérations régionales et des tensions entre " pouvoir fédéral » et «pouvoirs régionaux » rendent le débat assez brûlant.

L'article de Dominique Demelenne est consacré à l'introduction au Paraguay d'un programme d'éducation bilingue (espagnol-guarani). Seul pays d'Amérique latine où la majorité de la population pratique encore une langue indigène sur le territoire national, le Paraguay constitue une sorte de laboratoire de prise en compte de la diversité linguistique dans la région. Le texte montre les péripéties pédagogiques et opérationnelles de la mise en œuvre d'une réforme cherchant à valoriser l'enseignement de langues présentes dans la culture nationale. Ce processus produit des effets différents de ce que l'on recherchait. Les parents semblent hésitants face à cette valorisation récente du guarani et les enseignants sont confrontés aux difficultés d'opérationnalisation du guarani standard dans la classe. Dans cette expérience locale, le bilinguisme apparaît alors à l'école comme un exercice de style ne faisant pas toujours sens pour les acteurs scolaires.

Dans sa contribution, Beñat Muguruza présente la situation de la scolarisation en langue basque dans les parties espagnoles de l'espace où le basque est présent. Le contraste est souligné entre les options propres à la communauté autonome du Pays basque, où trois types de curriculum sont offerts, faisant une place plus ou moins importante à l'enseignement du/en basque, et la communauté forale de Navarre où, selon les zones considérées d'ouest en est, le basque est de moins en moins reconnu et l'instruction en basque moins offerte au choix 
des familles. Dans la communauté autonome du Pays basque, l'option qui prévoit une instruction scolaire entièrement en basque est celle qui a le plus progressé, au détriment notamment de celle où l'enseignement est assuré en espagnol et où le basque n'est que langue enseignée. Exemple particulier de ce que le volontarisme politique régional, appuyé par un soutien populaire majoritaire, peut réaliser, même s'agissant d'une langue dont la diffusion et la valeur d'usage ne sont que locaux?

L'article d'Aishah Binte Mohamad Kassim met en évidence la singularité de la situation linguistique à Singapour, pays où cohabitent quatre langues officielles (malais, chinois mandarin, tamoul, anglais) surtout parlées comme langues secondes hors de leurs communautés respectives. Bien que le malais soit considéré symboliquement comme la "langue nationale », les autorités au pouvoir ont préféré promouvoir l'usage de l'anglais dès la séparation du pays de la Malaisie. Par conséquent, l'anglais semble s'imposer non seulement au travail mais de plus en plus à la maison. Malgré une politique de l'État basé sur l'égalité entre les langues officielles d'enseignement, de plus en plus de parents, quelles que soient leurs langues familiales d'origine, choisissent d'envoyer leurs enfants à l'école de langue anglaise.

Le texte de Jean-Claude Beacco porte sur l'action du Conseil de l'Europe en matière de politique des langues dans l'enseignement. L'auteur montre que malgré les moyens d'action limités d'une organisation internationale comme le Conseil de l'Europe comparés à ceux des États, cette institution a pu mettre en exergue de "grands principes» traduits par les États en termes adaptés à leur contexte spécifique. D’une part, le Conseil de l'Europe propose des instruments, dont le plus connu est le Cadre européen commun de référence pour les langues (CECR). D'autre part, il agit aussi à travers d'autres moyens, plus politiques, comme des conventions-cadres et des chartes qui visent la protection des minorités nationales ou la promotion des langues régionales ou minoritaires. Loin de se substituer à l'État-nation en matière de politique linguistique, le Conseil de l'Europe influence et oriente les choix par le dialogue politique et les innovations.

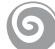

La comparaison, entreprise dans ce numéro, des termes du débat sur les langues d'enseignement est porteuse de certaines convergences et de questionnements qu'il est utile de dégager.

En premier lieu, le choix de la langue ou des langues d'instruction est un thème politique par excellence, qui dépasse largement le cadre de l'éducation. Il renvoie à la répartition du pouvoir entre les différentes échelles institutionnelles (national, régional, local), groupes culturels et acteurs divers. Une démocratisation ou ouverture de l'espace politique se manifeste habituellement aussi par un débat sur les langues d'enseignement. 
En second lieu, quand le débat ou les décisions politiques arrivent au niveau du système éducatif, ils se traduisent souvent par une remise en question de la gouvernance traditionnelle de ce système. Trois acteurs entrent en force dans ce processus :

- les pouvoirs politiques régionaux qui peuvent, dans le cadre d'une politique de décentralisation, gagner de nouvelles compétences et s'en saisir parfois pour contrer des orientations nationales;

- les parents qui, par des stratégies multiformes, sont susceptibles de contourner les orientations politiques nationales ou régionales en matière de choix de langues d'enseignement;

- enfin, les organisations internationales qui ont également une certaine capacité d'influence pour infléchir la gouvernance traditionnelle.

En troisième lieu, quand les options multiples en matière de langues d'enseignement arrivent sur le terrain scolaire, d'autres enjeux apparaissent. On peut en relever trois principaux. Le premier concerne la tension entre, d'un côté, des systèmes éducatifs de plus en plus centrés sur les résultats d'apprentissage et, de l'autre, la prise en compte de la diversité linguistique des élèves. Le deuxième renvoie au risque que constitue une fragmentation du système éducatif en plusieurs réseaux à plusieurs vitesses s'adressant à des publics ciblés. Le troisième enjeu, plus pédagogique et didactique que politique, concerne les prérequis qu'une langue doit remplir pour se convertir en une langue d'enseignement. On peut penser en particulier à une certaine standardisation, à une production significative de documents écrits et à une présence dans tous les espaces sociaux et pas seulement à l'école (radio, télévision et presse écrite).

\section{Bibliographie}

BRAY M., KOO R. (2004) : "Postcolonial patterns and paradoxes: Language and education in Hong Kong and Macao ", Comparative Education, 40(2), 215-239.

BRAY T. M. (2009) : Confronting the shadow education system: What government policies for what private tutoring? (p. 1-132), United Nations Educational, Scientific and Cultural Organization; International Institute for Educational Planning.

BROCK-UTNE B. (2007) : "Language of instruction and student performance: New insights from research in Tanzania and South Africa", International Review of Education, 53(5-6), 509-530.

CISSE M. (2005) : «Langues, État et société au Sénégal », Sudlangues, 5, 99-133.

CALVET L.-J., CALVET A. (2013) : Les confettis de Babel. Diversité linguistique et politique des langues, Paris : Écriture.

DESAI Z. (2013) : «Local languages: Good for the informal marketplace but not for the formal classroom ? », Education as Change, 17(2), 193-207.

GRANDGUILLAUME G. (2009) : "Langue maternelle et langue d'enseignement: comment les concilier?", dans Laroussi F. (ed.), Langues, identités et insularité: regards sur Mayotte (107-115). Rouen : Publications des universités de Rouen et du Havre. 
LIPPI-GREEN R. (1997) : English with an accent: language, ideology and discrimination in the United States, London : Routledge.

MENKEN K., GARCÍA O. (eds.), (2010) : Negotiating language education policies: Educators as policymakers, London : Routledge.

PHYAK P. (2013) : "Language ideologies and local languages as the medium-ofinstruction policy: A critical ethnography of a multilingual school in Nepal ", Current Issues in Language Planning, 14(1), 127-143.

SKATTUM I. (2010) : "L'introduction des langues nationales dans le système éducatif au Mali : objectifs et conséquences ", Journal of Language Contact, THEMA, 3, 247-270.

TOLLEFSON J. W. (2002) : Language policies in education: Critical issues. Mahwah, NJ : LEA. 